\title{
Quantum Walk Coupled to Environment
}

\author{
Guangbo Yang, Caishi Wang, Yan Luo, Yanyan Wang, Xueqi Nan
}

Abstract-In this paper, we considered quantum walk coupled to environment by introducing the concept of diamond product. We examined its basic properties and its unitary representation was obtained.

Index Terms-Quantum walk; Quantum Bernoulli Noises; Diamond Product; Unitary Operator

\section{INTRODUCTION}

As quantum analogs of classical random walks, quantum walks were introduced almost three decade ago, which have found wide application in quantum information, quantum computing and many other fields [1]. In 2016, Wang and Ye introduced a quantum walk model in terms of quantum Bernoulli noises [2]. They said that at some special initial states the walk has the same limit probability distribution as the classical random walk. Lauri Lehman studied the emergence of the mixing process in quantum walks [5] in 2014 and he pointed that the walker becomes entangled with environment when the emergence of the mixing process in quantum walk.

In this paper, we considered quantum walk coupled to environment by introducing the concept of diamond product. We examined its basic properties, and its unitary representation was obtained.

\section{PRELIMINARIES}

In this section, we briefly recall some notions and results for quantum Bernoulli noises. For details, see [2-4] and references therein.

Throughout this paper, $\mathbf{Z}$ always denotes the set of all integers, while $¥$ means the set of all nonnegative integers.

Let $W=\{-1,1\}^{¥}$ be the set of all mappings

$w: ¥ \mathrm{a} \quad\{-1,1\}$, and $\left(x_{n}\right)_{n^{3} 0}$ the sequences of canonical projections on given by

$$
x_{n}(w)=w(n) \quad w \hat{\mathrm{I}} \mathrm{W}
$$

Let $\mathrm{G}$ the finite power set of $¥$, namely,

$$
\mathrm{G}=\{s \mid s \text { Ì } ¥ \text { and } \# s<¥\} \text {, }
$$

Where \# $s$ denotes the cardinality of $s$ as a set. Let $\mathrm{F}$ be the $s$-field on W generated by the sequences $\left(x_{n}\right)_{n^{3} 0}$. Let

Guangbo Yang, School of Mathematics and Statistics, Northwest Normal University, Lanzhou, China, Mobile, 86-15769391379.

Caishi Wang, School of Mathematics and Statistics, Northwest Normal University, Lanzhou, China.

Yan Luo, School of Mathematics and Statistics, Northwest Normal University, Lanzhou, China.

Yanyan Wang, School of Mathematics and Statistics, Northwest Normal University, Lanzhou, China.

Xueqi Nan, School of Mathematics and Statistics, Northwest Normal University, Lanzhou, China. $\left(p_{n}\right)_{n^{3} 0}$ be a given sequences of positives numbers with the property that $0<p_{n}<1$ for all $n^{3} 0$. Then there exists a unique probability measure $\mathrm{P}$ on $\mathrm{F}$ such that

$$
\mathrm{Po}\left(z_{n_{1}}, z_{n_{2}}, \mathrm{~L}, z_{n_{k}}\right)^{-1}\left\{\left(\grave{\mathrm{o}}_{1}, \grave{\mathrm{o}}_{2}, \mathrm{~L}, \grave{\mathrm{o}}_{k}\right)\right\}=\mathrm{O}_{j=1}^{\mathrm{K}} p_{j}^{\frac{1+\grave{\mathrm{o}}_{j}}{2}} q_{j}^{\frac{1-\grave{\mathrm{o}}_{j}}{2}},
$$

for $n_{j} \hat{\mathrm{I}} ¥, \grave{\mathrm{o}}_{j} \hat{\mathrm{I}}\{-1,1\}(1 £ j £ k)$ with $n_{i}{ }^{1} n_{j}$ when $i^{1} \quad j$ and $k \hat{\mathrm{I}} ¥$ with $k^{3} 1$. Thus one has a probability measure space $(\mathrm{W}, \mathrm{F}, \mathrm{P})$, which is referred to as the Bernoulli space and random variables on it are known as Bernoulli functionals.

Let $Z=\left(Z_{n}\right)_{n^{3} 0}$ be the sequences of Bernoulli functionals defined by

$$
Z_{n}:=\frac{z_{n}+q_{n}-p_{n}}{2 \sqrt{p_{n} q_{n}}}, \quad n^{3} 0,
$$

where $q_{n}=1-p_{n}$. Clearly $Z=\left(Z_{n}\right)_{n^{3} 0}$ is an independent sequence of random variables on the probability measure space $(\mathrm{W}, \mathrm{F}, \mathrm{P})$.

Let $\mathrm{h}$ be the space of square integrable complex-valued random variables on $(\mathrm{W}, \mathrm{F}, \mathrm{P})$, namely

$$
\mathrm{h}=L^{2}(\mathrm{~W}, \mathrm{~F}, \mathrm{P}) \text {. }
$$

We denote by $\langle x\rangle$ the inner product of $h$, and by $\| \times x$ the corresponding norm. It is known that $Z$ has the orthonormal basis $\left\{Z_{s} \mid s\right.$ î $\left.G\right\}$ where $Z_{Æ}=1$ and

$$
Z_{s}=\tilde{\mathrm{O}} Z_{i}, \quad s \text { Î G } s^{1} \nRightarrow
$$

which shows that $\mathrm{h}$ is an infinite dimensional space.

\section{MAIN RESULTS}

In this section, we consider quantum walk coupled to environment and examine its basic properties.

Let $h$ be the space of square integrable Bernoulli functionals and $\mathrm{J}: £^{2} \ddot{A} \mathrm{~h} \AA \mathrm{h}$ a unitary isomorphism. Such a unitary isomorphism $\mathrm{J}$ does exist since $\mathrm{h}$ is a separable and infinite-dimensional Hilbert space over the complex number field $£$.

Definition 1. Let $A$ be an operator on $£^{2}$ and $B$ a bounded operator on $\mathrm{h}$. Then their diamond product $A$ à $B$ is defined as $A$ à $B=\mathrm{J}(A \ddot{\mathrm{A}} B) \mathrm{J}^{-1}$. 


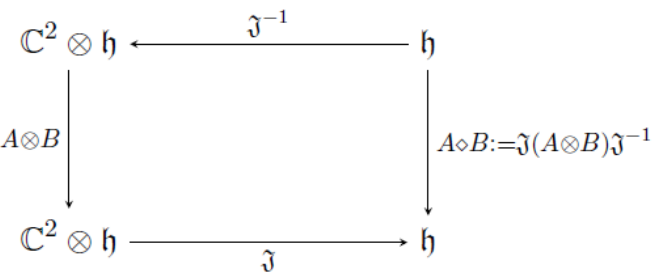

Figure 1 Diamond Product

Clearly, for an operator $A$ on $£^{2}$ and a bounded operator $B$ on $\mathrm{h}$, their diamond product $A$ à $B$ is an bounded operator on $\mathrm{h}$.

This provides an alternative way to make products between operators on $£^{2}$ and bounded operators on $h$.

Proposition 1. The diamond product admits the following algebraic properties

(1) $A$ à $B=0$ whenever $A=0$ or $B=0$;

(2) $I_{\mathfrak{f}^{2}} \grave{\mathrm{a}} I_{\mathrm{h}}=I_{\mathrm{h}}$, where $I_{\mathfrak{f}^{2}}$ and $I_{\mathrm{h}}$ denote identity operators on $£^{2}$ and $\mathrm{h}$, respectively;

(3) $(A \text { à } B)^{*}=A^{*}$ à $B^{*}$;

(4) $\left(A_{1}\right.$ à $\left.B_{1}\right)\left(A_{2}\right.$ à $\left.B_{2}\right)=\left(A_{1} A_{2}\right)$ à $\left(B_{1} B_{2}\right)$;

(5) $A$ à $\left(B_{1}+B_{2}\right)=A$ à $B_{1}+A$ à $B_{2}$,

$\left(A_{1}+A_{2}\right)$ à $B=A_{1} \grave{a} B+A_{2} \grave{a ̀ ~} B ;$

(6) $(a A)$ à $B=A$ à $(a B)=a(A$ à $B)$, where $a$ is any complex number;

(7) $A$ à $B$ is unitary whenever both $A$ and $B$ are unitary, where $A, A_{1}, A_{2}$ are operators on $£^{2}$ and $B, B_{1}, B_{2}$ are bounded operators on $\mathrm{h}$.

Proof. These are trivial results, so we only verify (2), (3) and (4) in here, and the rest of the results can be verified in a similar way. Let $A, A_{1}, A_{2}$ are operators on $£^{2}$ and $B$, $B_{1}, B_{2}$ are bounded operators on $\mathrm{h}$.

(2) For every $q \hat{\mathrm{I}} \mathrm{h}$, we have

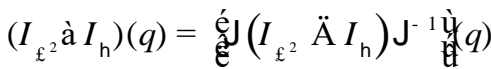

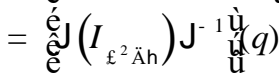

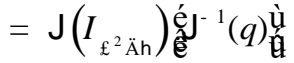

$$
\begin{aligned}
& =\mathrm{JJ}^{-1}(q)=q \text {, }
\end{aligned}
$$

namely $I_{\mathrm{f}^{2}}$ à $I_{\mathrm{h}}=I_{\mathrm{h}}$.

(3) It is noted that $\mathrm{J}$ is a unitary isomorphism operator form $£^{2} \ddot{A} \mathrm{~h}$ to $\mathrm{h}$, so we have $\mathrm{J}^{*} \mathrm{~J}=\mathrm{JJ}^{*}=I$, namely $\mathrm{J}^{-1}=\mathrm{J}^{*}$. By simple calculation, we get

$$
\begin{aligned}
& (A \text { à } B)^{*}=\left(\mathrm{J}(A \ddot{A} B) \mathrm{J}^{-1}\right)^{*} \\
& =\left(\mathrm{J}^{-1}\right)^{*}(A \ddot{A} B)^{*} \mathrm{~J}^{*} \\
& =\left(\mathrm{J}^{*}\right)^{*}\left(A^{*} \ddot{\mathrm{A}} B^{*}\right) \mathrm{J}^{-1} \\
& =\mathrm{J}\left(A^{*} \ddot{\mathrm{A}} B^{*}\right) \mathrm{J}^{-1} \\
& =A^{*} \mathrm{à} B^{*} .
\end{aligned}
$$

(4) From the feature of the tensor product, we get

$$
\begin{aligned}
& \left(A_{1} \grave{a} B_{1}\right)\left(A_{2} \text { à } B_{2}\right)
\end{aligned}
$$

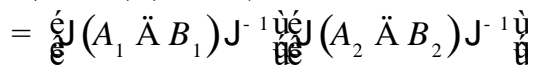

$$
\begin{aligned}
& =\mathrm{J}\left(A_{1} \ddot{\mathrm{A}} B_{1}\right) \mathrm{J}^{-1} \mathrm{~J}\left(A_{2} \ddot{\mathrm{A}} B_{2}\right) \mathrm{J}^{-1} \\
& =\mathrm{J}\left(A_{1} \ddot{\mathrm{A}} B_{1}\right)\left(A_{2} \ddot{\mathrm{A}} B_{2}\right) \mathrm{J}^{-1} \\
& \left.=\mathrm{J} A_{1} A_{2}\right) \ddot{\mathrm{A}}\left(B_{1} B_{2}\right) \mathrm{ù}^{-1} \\
& =\left(A_{1} A_{2}\right) \grave{a ̀ ~}\left(B_{1} B_{2}\right) \text {, }
\end{aligned}
$$

the conclusion is proved.

Definition 2. A pair $(P, Q)$ of operators on $£^{2}$ is called a coin operator pair if the sum $P+Q$ is unitary and

$$
P^{*} Q=P Q^{*}=Q^{*} P=Q P^{*}=0 .
$$

It is worth to notify that for a coin operator pair $(P, Q)$ on $£^{2}$, one always has the following useful relations

$$
P^{*} P+Q^{*} Q=I, \quad P P^{*}+Q Q^{*}=I .
$$

In fact, for the sum $P+Q$ is unitary, we have

$$
\begin{aligned}
I_{\mathcal{E}^{2}} & =(P+Q)^{*}(P+Q)=\left(P^{*}+Q^{*}\right)(P+Q) \\
& =P^{*} P+P^{*} Q+Q^{*} P+Q^{*} Q \\
& =P^{*} P+Q^{*} Q,
\end{aligned}
$$

In a similar way, we get $P P^{*}+Q Q^{*}=I$.

Proposition 2. Let $P$ and $Q$ be operators on $£^{2}$ satisfying that $P+Q$ is unitary and

$$
P^{*} Q=P Q^{*}=Q^{*} P=Q P^{*}=0 .
$$

Assume further that $E_{1}, E_{2}$ are unitary operators on $\mathrm{h}$. Then $P$ à $E_{1}+Q$ à $E_{2}$ is a unitary operator on h, and moreover it holds true that

$$
\begin{aligned}
\left(P \text { à } E_{1}\right)^{*}\left(Q \text { à } E_{2}\right) & =\left(P \text { à } E_{1}\right)\left(Q \text { à } E_{2}\right)^{*} \\
& =\left(Q \text { à } E_{2}\right)^{*}\left(P \text { à } E_{1}\right) \\
& =\left(Q \text { à } E_{2}\right)\left(P \text { à } E_{1}\right)^{*}=0,
\end{aligned}
$$

where 0 means the null operator (zero operator) on $\mathrm{h}$.

Proof. Since $P+Q$ is unitary, denoting by $I_{\mathfrak{f}^{2}}$ the identity operator on $£^{2}$, we have

$$
\begin{aligned}
I_{\mathfrak{f}^{2}} & =(P+Q)^{*}(P+Q) \\
& =\left(P^{*}+Q^{*}\right)(P+Q) \\
& =P^{*} P+P^{*} Q+Q^{*} P+Q^{*} Q,
\end{aligned}
$$

which together with $P^{*} Q=Q^{*} P=0$ gives $P^{*} P+Q^{*} Q=I_{\mathfrak{f}^{2}}$. Similarly, we have $P P^{*}+Q Q^{*}=I_{\mathfrak{f}^{2}}$.

By straightforward calculation, we get

$$
\begin{aligned}
& \left(P \text { à } E_{1}+Q \text { à } E_{2}\right)^{*}\left(P \text { à } E_{1}+Q \text { à } E_{2}\right) \\
= & \left(P^{*} \text { à } E_{1}^{*}+Q^{*} \text { à } E_{2}^{*}\right)\left(P \text { à } E_{1}+Q \text { à } E_{2}\right) \\
= & \left(P^{*} \text { à } E_{1}^{*}\right)\left(P \text { à } E_{1}\right)+\left(P^{*} \text { à } E_{1}^{*}\right)\left(Q \grave{a ̀ ~} E_{2}\right) \\
& +\left(Q^{*} \text { à } E_{2}^{*}\right)\left(P \text { à } E_{1}\right)+\left(Q^{*} \text { à } E_{2}^{*}\right)\left(Q \grave{a ̀ ~} E_{2}\right) \\
= & \left(P^{*} P\right) \grave{a}\left(E_{1}^{*} E_{1}\right)+\left(P^{*} Q\right) \grave{a ̀ ~}\left(E_{1}^{*} E_{2}\right) \\
& +\left(Q^{*} P\right) \text { à }\left(E_{2}^{*} E_{1}\right)+\left(Q^{*} Q\right) \text { à }\left(E_{2}^{*} E_{2}\right) \\
= & \left(P^{*} P\right) \text { à } I_{\mathrm{h}}+\left(Q^{*} Q\right) \text { à } I_{\mathrm{h}} \\
= & \left(P^{*} P+Q^{*} Q\right) \text { à } I_{\mathrm{h}} \\
= & I_{\epsilon^{2}} \grave{a ̀ ~} I_{\mathrm{h}} \\
= & I_{\mathrm{h}} .
\end{aligned}
$$


Similarly we can get

$$
\left(P \text { à } E_{1}+Q \text { à } E_{2}\right)\left(P \text { à } E_{1}+Q \text { à } E_{2}\right)^{*}=I_{\mathrm{h}} \text {. }
$$

Thus $P$ à $E_{1}+Q$ à $E_{2}$ is a unitary operator on $\mathrm{h}$. Now we show that the four equalities in (7) hold.

In fact, by using Proposition 1. and the property of $P^{*} Q=0$, we can come to

$$
\begin{aligned}
& \left(P \text { à } E_{1}\right)^{*}\left(Q \text { à } E_{2}\right)=\left(P^{*} \text { à } E_{1}^{*}\right)\left(Q \text { à } E_{2}\right) \\
= & \left(P^{*} Q\right) \text { à }\left(E_{1}^{*} E_{2}\right)=0 \text { à }\left(E_{1}^{*} E_{2}\right)=0 .
\end{aligned}
$$

In a similar way, we can also show that the rest three equalities hold.

Remark 1. The implication of Proposition 2 lies in the following observations. Let $E_{1}, E_{2}$ be unitary operators on h. Then, according to Proposition 2, ( $P$ à $E_{1}, Q$ à $\left.E_{2}\right)$ is a coin operator pair on $\mathrm{h}$ when $(P, Q)$ is a coin operator pair on $£^{2}$.

Theorem 1. Let $(P, Q)$ be a coin operator pair on $£^{2}$ and $E_{1}, E_{2}$ be unitary operators on the space of square integrable Bernoulli functionals $\mathrm{h}$. For all F $\hat{\mathrm{I}} l^{2}(\phi, \mathrm{h})$, we define $\mathrm{Y}: \not \AA \mathrm{h}$ as follows

$$
\begin{aligned}
\mathrm{Y}(x):= & \left(P \text { à } E_{1}\right) \mathrm{F}(x-1) \\
& +\left(Q \text { à } E_{2}\right) \mathrm{F}(x+1), \quad x \text { Î } \mathbf{Z},
\end{aligned}
$$

then $\mathrm{Y}$ I $l^{2}(\mathbf{Z}, \mathrm{h})$ and $\|\mathrm{Y}\|_{l^{2}(\mathbf{z}, \mathrm{h})}=\|\mathrm{F}\|_{l^{2}(\mathbf{z}, \mathrm{h})}$.

Proof. We first verify $\|\mathrm{Y}\|_{l^{2}(\mathbf{z}, \mathrm{h})}=\|\mathrm{F}\|_{l^{2}(\mathbf{z}, \mathrm{h})}$.

For F î $l^{2}(\phi, h)$, we have

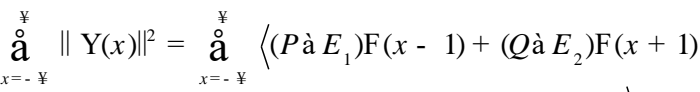

$$
\begin{aligned}
& \left.\left(P \text { à } E_{1}\right) \mathrm{F}(x-1)+\left(Q \text { à } E_{2}\right) \mathrm{F}(x+1)\right\rangle \\
& =\stackrel{\ddagger}{\mathrm{a}} \mathrm{a} \mathrm{F}(x-1),\left(P \text { à } E_{1}\right)^{*}\left(P \text { à } E_{1}\right) \mathrm{F}(x-1) \tilde{n} \\
& +\stackrel{\sharp}{\circ} \mathrm{a} \quad \mathrm{a} F(x-1),\left(P \text { à } E_{1}\right)^{*}\left(Q \grave{a ̀} E_{2}\right) \mathrm{F}(x+1) \tilde{n} \\
& \underset{x=-¥}{\neq} \\
& + \text { å̀ } \mathrm{a} \mathrm{F}(x+1),\left(Q \mathrm{à} E_{2}\right)^{*}\left(P \mathrm{à} E_{1}\right) \mathrm{F}(x-1) \tilde{n}
\end{aligned}
$$

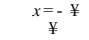

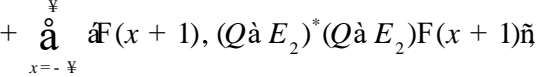

according to formula (8), we know

$$
\begin{aligned}
& \stackrel{\ddagger}{\stackrel{\ddagger}{\mathrm{a}}}\|\mathrm{Y}(x)\|^{2}
\end{aligned}
$$

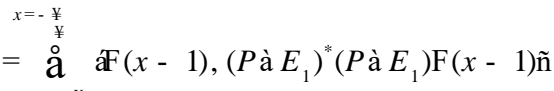

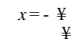

$$
\begin{aligned}
& +\stackrel{\text { å }}{\mathrm{a}} \mathrm{a} \mathrm{F}(x+1),\left(Q \mathrm{à} E_{2}\right)^{*}\left(Q \mathrm{à} E_{2}\right) \mathrm{F}(x+1) \tilde{n} \\
& \begin{array}{c}
x=-¥ \\
¥ \\
0
\end{array} \\
& =\stackrel{\stackrel{p}{\circ}}{\mathrm{a}} \mathrm{a} \mathrm{F}(x),\left(P \text { à } E_{1}\right)^{*}\left(P \text { à } E_{1}\right) \mathrm{F}(x) \tilde{\mathrm{n}} \\
& x=-\underset{z}{\sharp} \\
& +\stackrel{¥}{\mathrm{a}} \mathrm{a} \mathrm{F}(x),\left(Q \text { à } E_{2}\right)^{*}\left(Q \text { à } E_{2}\right) \mathrm{F}(x) \tilde{n} \\
& \underset{\substack{x=-* \\
\ddagger}}{\sharp}
\end{aligned}
$$

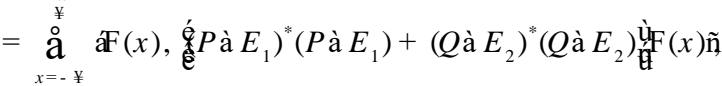

which together with

$$
\begin{aligned}
& \left(P \text { à } E_{1}\right)^{*}\left(P \text { à } E_{1}\right)+\left(Q \text { à } E_{2}\right)^{*}\left(Q \text { à } E_{2}\right) \\
= & \left(P^{*} \text { à } E_{1}^{*}\right)\left(P \text { à } E_{1}\right)+\left(Q^{*} \text { à } E_{2}^{*}\right)\left(Q \text { à } E_{2}\right) \\
= & \left(P^{*} P\right) \text { à }\left(E_{1}^{*} E_{1}\right)+\left(Q^{*} Q\right) \text { à }\left(E_{2}^{*} E_{2}\right) \\
= & \left(P^{*} P\right) \text { à } \mathrm{I}_{\mathrm{h}}+\left(Q^{*} Q\right) \text { à } \mathrm{I}_{\mathrm{h}} \\
= & \left(P^{*} P+Q^{*} Q\right) \text { à } \mathrm{I}_{\mathrm{h}} \\
= & \mathrm{I}_{\mathfrak{E}^{2}} \text { à } \mathrm{I}_{\mathrm{h}}=\mathrm{I}_{\mathrm{h}},
\end{aligned}
$$

we have

$$
\begin{aligned}
& \stackrel{\ddagger}{\stackrel{\sharp}{a}}\|\mathrm{Y}(x)\|^{2}
\end{aligned}
$$

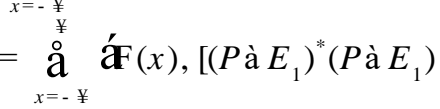

$$
\begin{aligned}
& \left.+\left(Q \text { à } E_{2}\right)^{*}\left(Q \text { à } E_{2}\right)\right] \mathrm{F}(x) \tilde{n} \\
& =\underset{x=-¥}{\stackrel{\ddagger}{\mathbf{a}}} \mathbf{a} \mathbf{F}(x), \mathrm{F}(x) \tilde{\mathbf{n}}=\underset{x=-¥}{\stackrel{\ddagger}{\mathrm{a}}}\|\mathrm{F}(x)\|^{2},
\end{aligned}
$$

namely $\|\mathrm{Y}\|_{l^{2}(\mathbf{z}, \mathrm{h})}=\|\mathrm{F}\|_{l^{2}(\mathbf{z}, \mathrm{h})}$. Together with equation (9) and F î $l^{2}(\phi, h)$, we can know Y î $l^{2}(\mathbf{Z}, \mathrm{h})$.

Definition 3. The quantum walk on $\varnothing$ acting with environment $h$ has the following features

(1) The walk takes $l^{2}(\mathbf{Z}, \mathbf{h})$ as its state space and its states are represented by unit vectors in $l^{2}(\mathbf{Z}, \mathrm{h})$;

(2) The walk is of discrete times and its time evolution satisfies $\mathrm{F}_{n+1}(x)=\left(P\right.$ à $\left.E_{1}\right) \mathrm{F}_{n}(x-1)$

$$
+\left(Q \mathrm{à} E_{2}\right) \mathrm{F}_{n}(x+1), \quad x \hat{\mathrm{I}} \not, n^{3} 0,
$$

where $\mathrm{F}_{n}$ denotes the state of the walk at time $n^{3} 0$, $(P, Q)$ is a coin operator pair on $£^{2}$, and $E_{1}, E_{2}$ are random unitary operators on $\mathrm{h}$.

Here a random unitary operator on $h$ means a mapping form some probability space to the group of unitary operators on $\mathrm{h}$ that satisfies certain measurable conditions.

Let $\mathrm{F}_{n}$ be the state of the walk at time $n^{3} 0$. Then, the function $x$ a $\left\|\mathrm{F}_{n}(x)\right\|^{2}$ makes a probability distribution on $\varnothing$. We call the function the probability distribution of the walk at time $n^{3} 0$.

It can be shown that the quantity $\left\|\mathrm{F}_{n}(x)\right\|^{2}$ is just the probability of finding the walker at position $x$ and time $n$.

The physical meaning of the above walk lies in the observations below. As the usual models, the walk's internal degrees of freedom are described by the coin operator pair $(P, Q)$ on $£^{2}$, while its conditioned shifts are represented by the shift operator $S$ and its adjoint $S^{*}$ on $l^{2}(\notin)$. The operators $E_{1}$ and $E_{2}$ on $\mathrm{h}$ just play the role of environment.

Theorem 2. Let $n^{3} 0$, then there exists a unitary operator $\mathrm{U}: l^{2}(\varnothing, \mathrm{h}){ }^{\circledR} l^{2}(\varnothing, \mathrm{h})$ such that 


\section{Quantum Walk Coupled to Environment}

$$
\begin{aligned}
{[\mathrm{UF}](x)=} & \left(P \text { à } E_{1}\right) \mathrm{F}(x-1) \\
& +\left(Q \text { à } E_{2}\right) \mathrm{F}(x+1), \quad \text { "F î } l^{2}(\phi, h), x \text { Î } \phi,
\end{aligned}
$$

moreover, its adjoint $\mathrm{U}^{*}$ satisfies

$$
\begin{aligned}
{\left[\mathrm{U}^{*} \mathrm{~F}\right](x)=} & \left(Q \mathrm{à} E_{2}\right)^{*} \mathrm{~F}(x-1) \\
& +\left(P \text { à } E_{1}\right)^{*} \mathrm{~F}(x+1), \quad \text { "F Î } l^{2}(\phi, \mathrm{h}), x \text { I } \phi .
\end{aligned}
$$

Proof. For each F î $l^{2}(\notin, h)$, we define the function $\mathrm{Y}_{\mathrm{F}}: \not \circledast \mathrm{h}$ as

$$
\mathrm{Y}_{\mathrm{F}}(x):=\left(P \text { à } E_{1}\right) \mathrm{F}(x-1)+\left(Q \text { à } E_{2}\right) \mathrm{F}(x+1), \quad x \hat{\mathrm{I}} \not,
$$

by Theorem 1 we have $\mathrm{Y}_{\mathrm{F}}(x) \hat{\mathrm{I}} l^{2}(\mathbf{Z}, \mathrm{h})$ and

$\left\|\mathrm{Y}_{\mathrm{F}}\right\|_{l^{2}(\mathbf{z}, \mathrm{h})}=\|\mathrm{F}\|_{l^{2}(\mathbf{z}, \mathrm{h})}$. Thus we can define an isometric operator $\mathrm{U}: l^{2}(\varnothing, \mathrm{h}){ }^{\circledR} l^{2}(\varnothing, \mathrm{h})$ such that

$$
\mathrm{U}=\mathrm{Y}_{\mathrm{F}}, \quad \mathrm{F} \hat{\mathrm{I}} l^{2}(\phi, \mathrm{h}),
$$

Which means the $\mathrm{U}$ satisfies (11).

Next, we consider $\mathrm{U}^{*}$ the adjoint of $\mathrm{U}$. Let $\mathrm{F} \hat{\mathrm{I}} l^{2}(\not, \mathrm{h})$, then for any $x \hat{\mathrm{I}} \mathbf{Z}, s \hat{\mathrm{I}} \mathrm{G}$, we define a function $\mathrm{F}^{(s)} \hat{\mathrm{I}} l^{2}(\mathbf{Z}, \mathrm{h})$ such that

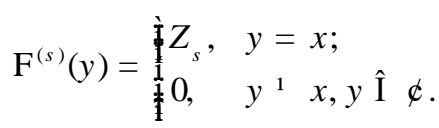

which gives

$$
\begin{aligned}
& \left\langle\mathrm{U}^{*}(x), Z_{s}\right\rangle=\left\langle\mathrm{U}^{*} \mathrm{~F}, \mathrm{~F}^{(s)}\right\rangle_{l^{2}(\dot{\phi}, \mathrm{h})} \\
& =\left\langle\mathrm{F}, \mathrm{UF}^{(s)}\right\rangle_{l^{2}(\dot{\phi}, \mathrm{h})}=\stackrel{\ddagger}{\stackrel{\ddagger}{\mathrm{a}}}\left\langle\mathrm{F}(y), \mathrm{UF}^{(s)}(y)\right\rangle \\
& =\underset{y=-\neq}{\stackrel{\ddagger}{\circ}}\left\langle\mathrm{F}(y),\left[\left(P \text { à } E_{1}\right) \mathrm{F}^{(s)}(y-1)+\left(Q \grave{a} E_{2}\right)\right] \mathrm{F}^{(s)}(y+1)\right\rangle \\
& =\underset{y=-\not}{\stackrel{\ddagger}{0}}\left\langle\mathrm{~F}(y),\left(P \text { à } E_{1}\right) \mathrm{F}^{(s)}(y-1)\right\rangle
\end{aligned}
$$

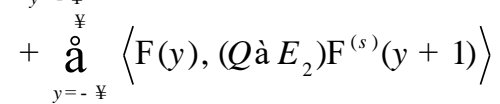

$$
\begin{aligned}
& =\left\langle\mathrm{F}(x+1),\left(P \text { à } E_{1}\right) \mathrm{F}^{(s)}(x)\right\rangle+\left\langle\mathrm{F}(x-1),\left(Q \text { à } E_{2}\right) \mathrm{F}^{(s)}(x)\right\rangle \\
& =\left\langle\mathrm{F}(x+1),\left(P \text { à } E_{1}\right) Z_{s}\right\rangle+\left\langle\mathrm{F}(x-1),\left(Q \grave{a ̀ ~} E_{2}\right) Z_{s}\right\rangle \\
& =\left\langle\left(P \text { à } E_{1}\right)^{*} \mathrm{~F}(x+1), Z_{s}\right\rangle+\left\langle\left(Q \grave{a} E_{2}\right)^{*} \mathrm{~F}(x-1), Z_{s}\right\rangle \\
& =\left\langle\left[\left(P \text { à } E_{1}\right)^{*} \mathrm{~F}(x+1)+\left(Q \text { à } E_{2}\right)^{*} \mathrm{~F}(x-1)\right], Z_{s}\right\rangle,
\end{aligned}
$$

Thus we have

$$
\begin{aligned}
{\left[\mathrm{U}^{*} \mathrm{~F}\right](x)=} & \left(Q \text { à } E_{2}\right)^{*} \mathrm{~F}(x-1) \\
& +\left(P \text { à } E_{1}\right)^{*} \mathrm{~F}(x+1) .
\end{aligned}
$$

Finally, we verify $U$ is a unitary operator. Since $U$ is a isometric operator, we just need to prove that $\mathrm{UU}^{*}=\mathrm{I}_{l^{2}(\dot{\phi}, \mathrm{h})}$. Let F Î $l^{2}(\varnothing, h)$, by straightforward calculation, we get

$$
\begin{aligned}
& {\left[\mathrm{UU}^{*} \mathrm{~F}\right](x) } \\
= & \mathrm{U}\left[\mathrm{U}^{*} \mathrm{~F}\right](x) \\
= & \left(P \text { à } E_{1}\right)\left[\mathrm{U}^{*} \mathrm{~F}\right](x-1)+\left(Q \grave{a} E_{2}\right)\left[\mathrm{U}^{*} \mathrm{~F}\right](x+1) \\
= & \left(P \text { à } E_{1}\right)\left[\left(Q \text { à } E_{2}\right)^{*} \mathrm{~F}(x-2)+\left(P \text { à } E_{1}\right)^{*} \mathrm{~F}(x)\right] \\
& +\left(Q \text { à } E_{2}\right)\left[\left(Q \grave{a} E_{2}\right)^{*} \mathrm{~F}(x)+\left(P \text { à } E_{1}\right)^{*} \mathrm{~F}(x+12)\right] \\
= & \left(P \text { à } E_{1}\right)\left(Q \text { à } E_{2}\right)^{*} \mathrm{~F}(x-2)+\left(P \text { à } E_{1}\right)\left(P \text { à } E_{1}\right)^{*} \mathrm{~F}(x) \\
& +\left(Q \text { à } E_{2}\right)\left(Q \text { à } E_{2}\right)^{*} \mathrm{~F}(x)+\left(Q \text { à } E_{2}\right)\left(P \text { à } E_{1}\right)^{*} \mathrm{~F}(x+2) \\
= & \left(P \text { à } E_{1}\right)\left(Q^{*} \text { à } E_{2}^{*}\right) \mathrm{F}(x-2)+\left(P \text { à } E_{1}\right)\left(P^{*} \text { à } E_{1}^{*}\right) \mathrm{F}(x) \\
& +\left(Q \text { à } E_{2}\right)\left(Q^{*} \text { à } E_{2}^{*}\right) \mathrm{F}(x)+\left(Q \text { à } E_{2}\right)\left(P^{*} \text { à } E_{1}^{*}\right) \mathrm{F}(x+2) \\
= & \left(P Q^{*}\right) \grave{a ̀ ~}\left(E_{1} E_{2}^{*}\right) \mathrm{F}(x-2)+\left(P P^{*}\right) \text { à }\left(E_{1} E_{1}^{*}\right) \mathrm{F}(x) \\
& +\left(Q Q^{*}\right) \text { à }\left(E_{2} E_{2}^{*}\right) \mathrm{F}(x)+\left(Q P^{*}\right) \text { à }\left(E_{2} E_{1}^{*}\right) \mathrm{F}(x+2),
\end{aligned}
$$

for $P Q^{*}=Q P^{*}=0$, thus

$$
\begin{aligned}
& \text { [UU* } \left.{ }^{*}\right](x) \\
& =\left(P Q^{*}\right) \mathrm{à}\left(E_{1} E_{2}^{*}\right) \mathrm{F}(x-2)+\left(P P^{*}\right) \text { à }\left(E_{1} E_{1}^{*}\right) \mathrm{F}(x) \\
& +\left(Q Q^{*}\right) \mathrm{à}\left(E_{2} E_{2}^{*}\right) \mathrm{F}(x)+\left(Q P^{*}\right) \text { à }\left(E_{2} E_{1}^{*}\right) \mathrm{F}(x+2) \\
& =\left(P P^{*}\right) \mathrm{à}\left(E_{1} E_{1}^{*}\right) \mathrm{F}(x)+\left(Q Q^{*}\right) \mathrm{à}\left(E_{2} E_{2}^{*}\right) \mathrm{F}(x) \\
& =\left(P P^{*}\right) \text { à } \mathrm{I}_{l^{2}(\phi, h)} \mathrm{F}(x)+\left(Q Q^{*}\right) \text { à } \mathrm{I}_{l^{2}(\phi, h)} \mathrm{F}(x) \\
& =\left[\left(P P^{*}\right) \text { à } \mathrm{I}_{l^{2}(\dot{\phi}, \mathrm{h})}+\left(Q Q^{*}\right) \text { à } \mathrm{I}_{l^{2}(\dot{k}, \mathrm{~h})} \mathbb{F}(x)\right. \\
& =\left[\left(P P^{*}+Q Q^{*}\right) \text { à } \mathrm{I}_{l^{2}(\dot{\phi}, \mathrm{h})} \mathrm{F}(x)\right. \\
& =\left[\mathrm{I}_{\mathfrak{f}^{2}} \text { à } \mathrm{I}_{l^{2}(\phi, h)} \mathbb{F}(x)\right. \\
& =\mathrm{I}_{l^{2}(\dot{x}, \mathrm{~h})} \mathrm{F}(x)=\mathrm{F}(x),
\end{aligned}
$$

where $x$ I $\mathbf{Z}$. Thus $\mathrm{UU}^{*} \mathrm{~F}=\mathrm{F}$, which together with the arbitrariness of $\mathrm{F}$ implies that $\mathrm{UU}^{*}=\mathrm{I}_{l^{2}(k, \mathrm{~h})}$, namely $\mathrm{U}$ is a unitary operator.

Corollary 1. Form theorem 2, we can know that, quantum walk has a unitary representation, more precisely

$$
\mathrm{F}_{n}=\mathrm{UF}_{n-1}=\mathrm{U}^{2} \mathrm{~F}_{n-2}=\mathrm{L}=\mathrm{U}^{n} \mathrm{~F}_{0}, \quad n^{3} 1,
$$

where $\mathrm{U}: l^{2}(\phi, \mathrm{h}) \circledR l^{2}(\phi, \mathrm{h})$ is the unitary operator defined by (11).

\section{ACKNOWLEDGMENT}

This work is supported by National Natural Science Foundation of China (Grant No. 11461061).

\section{REFERENCES}

[1] Venegas-Andraca, S.E., Quantum walks: A com-prehensive review, J Quantum Inf. Process. 2012, 11, 1015-1106.

[2] Wang, C.S.; Ye, X.J., Quantum walk in terms of quantum Bernoulli noises, J. Quantum Inf. Process. 2016, 15, 1897-1908.

[3] C. Wang, H. Chai, Y. Lu, Discrete-time quantum Bernoulli noises, J. Journal of Mathematical Physics, 2010, 51(5), 23.

[4] C. Wang, J. Zhang, Localization of quantum Bernoulli noises, J. Journal of Mathematical Physics, 2013, 54(10), 23-278.

[5] Lauri Lehman, Environment-induced mixing processes in quantum walks, J. International Journal of Quantum Information, 2014, 12(4), 1450021.

Guangbo Yang, School of Mathematics and Statistics, Northwest Normal University, Lanzhou, China, Mobile, 86-15769391379.

Caishi Wang, School of Mathematics and Statistics, Northwest Norma University, Lanzhou, China.

Yan Luo, School of Mathematics and Statistics, Northwest Normal University, Lanzhou, China.

Yanyan Wang, School of Mathematics and Statistics, Northwest Normal University, Lanzhou, China.

Xueqi Nan, School of Mathematics and Statistics, Northwest Normal University, Lanzhou, China. 